\title{
Thermodynamic Formulation of Living Systems and Their Evolution II
}

\author{
L. F. del Castillo1* ${ }^{*}$ L. F. Martínez-Manzo1, V. Levario-Diaz ${ }^{2,3}$ \\ ${ }^{1}$ Polymers Department, Institute of Research in Materials, Universidad Nacional Autónoma de México, \\ Mexico City, Mexico \\ ${ }^{2}$ Bristol Centre for Functional Nanomaterials, HH Wills Physics Laboratory, University of Bristol, Bristol, UK \\ ${ }^{3}$ School of Chemistry, University of Bristol, Bristol, UK \\ Email: *lfelipe@unam.mx, ferminmartinez@ciencias.unam.mx, vl14732@bristol.ac.uk
}

How to cite this paper: del Castillo, L.F., Martínez-Manzo, L.F. and Levario-Diaz, V. (2020) Thermodynamic Formulation of Living Systems and Their Evolution II. Journal of Modern Physics, 11, 1279-1300. https://doi.org/10.4236/jmp.2020.119080

Received: August 17, 2020

Accepted: September 5, 2020

Published: September 8, 2020

Copyright $\odot 2020$ by author(s) and Scientific Research Publishing Inc. This work is licensed under the Creative Commons Attribution International License (CC BY 4.0).

http://creativecommons.org/licenses/by/4.0/ (c) (i) Open Access

\begin{abstract}
In the previous paper [1], the application of the general thermodynamic theory was considered to biological systems. The nature of living matter has been presented from the mesoscopic to the macroscopic point of view, for different time scales (ontogenetic and phylogenetics). Herein, we continue with this application, and present three characteristics of life in the form of statements or postulates. The first characteristic describes the probability of survival against aging. In particular, the behaviour of life is shown as an independent mode of aging. The second characteristic refers to the adaptation of the species according to the environment. The relationship between the phenomenon of organic homeostasis and the origin of the clinical parameters that define health is highlighted. And finally, the third characteristic applies the principle of negentropy to describe evolution. A representative model is given as an example of each postulate.
\end{abstract}

\section{Keywords}

Thermodynamics of Biological Systems, Characteristics of Life, Adaptation, Surviving Probability, Biological Irreversibility, Negentropy

\section{Introduction}

Thermodynamics is one of the physical theories based on postulated structures, in which the phenomena of physical nature are described. Its tremendous impact is highlighted in biology to describe phenomena at macroscopic (organs and tissues), mesoscopic (cells and cell structures) and nanometric (DNA and proteins) scales. Thermodynamics is a highly applicable physical science because its axiomatic thermodynamics, initially proposed by Carathéodory in 1909 [2] and 
later developed by Callen in 1985 [3], detail the state of equilibrium (zeroth law), the conservation of energy in terms of work and heat (first law), the growth of entropy (second law) and zero entropy at zero temperature (third law).

Similarly, the thermodynamic formulation by means of a small set of axioms, we wish to support three postulates, that could well summarize a description of different aspects of biological systems. We approach each of these postulates as an outstanding characteristic of living beings.

The first characteristic of life defines the way to maintain and increase the living capability [4] using the probabilistic aspect as a form to represent biological entities. Within this characteristic, a dynamic model of life is presented through the living capability, which is expressed as the increase in the probability of individual survival over time of life. The probability of living is determined by the search of resources, optimization of travelled places, niche perfection, defence, and scape actions, among others. Adaptive learning allows a member of a species to adapt its behaviour in response to stimuli or actions, depending on whether the environment is temporally or spatially homogenous or heterogenous [5] [6]. This behavioural adaptation developed by learning, improves the probability of individual survival of sub-optimal members within a population and facilitates their genetic evolution [7] by compensating for genotypic deficiencies and acquiring higher fitness with respect to a new or current environment [6] [7].

The second characteristic refers to the adaptation in terms of achieving a maximum metabolic efficiency, considered as the minimum entropy production with a minimum energy consumption. The second characteristic of the biological evolution is centred on the origin and development of the function of homeostasis: the ability to maintain stable the internal conditions of living beings. The organic adaptation phenomenon is related to this ability [8], since it is associated with the development of an efficient and adequate metabolism to use and conserve the matter and energy elaborated from elements acquired from outside the environment (law of least effort). The fundamental part is the creation of control mechanisms, product of the interaction with the environment. Evolutionary pressure on living organisms has been associated with the creation of cybernetic mechanisms for the predicted physiological regulation of clinical parameters that define health.

The third characteristic is the evolutionary growth of new generations by accumulation of taxa. Each taxon is a proportion of the negentropy gained in the form of structure accumulation. Evolutionary biologists have observed that species come from other species due to evolution, and there are different phenotypic characters, such as morphology, behaviour, anatomical development, embryo, genetic and protein variation. Species present homologous similarities at all levels of the phenotype; therefore, the differences that we observe today between species are due to the new genetic variations that they have acquired over time [9]. To refer to the description of this evolutionary phenomenon, the concept of negentropy is presented here. 


\section{First Characteristic of Life: Individual Surviving versus Aging}

Among the dynamics of animal behaviour [10] are the conservation in the broadest sense: shelter from environmental hazards and risks to maintain good health, the ability to search and acquire food during the representative life stages, and establish reproduction actions, including group integration for self-protection [11]. The survival-seeking behaviour arises as an emergent function derived from the inherited genetic information, which is recognized as "adaptive learning".

Ethology [12] begins with the study of the behaviours exhibited by any species, an "ethogram" (a description of the main types of behaviour with their frequencies of appearance). This science was founded on the works of Konrad Lorenz, Nikolaas Tinbergen, and Karl von Frisch, who were jointly awarded the Nobel Prize in Physiology and Medicine in 1973. The causality of life and ontogeny are summarized as the "proximate mechanisms", considering learning behaviour as the cornerstone of modern ethology and demonstrating how behaviour impacts the survival and reproduction chances of animals.

The biological species that have lived in an environment for a long time show adaptive characteristics, consisting of a behaviour in which protection, reproduction and obtaining of resources for survival are made efficient. The behaviour is largely determined by genes [13] and influenced to fit into the environment in a short time. This development is recognized as learning which occurs throughout life, but it is more intense during the first experiences after the birth stage, where imprinting occurs according to the ethologist Konrad Lorentz [14]. After that stage, the basis of the vertebral structure for future behaviour [15] is formed [16]. Given the importance of this description [17] we summarize the first characteristic of life of biological systems [18] in the following statement,

"Given a biological species with a defined genetic pool (genotype) in a specific environment, the probability of individual survival is a function that depends on the monotonous growth of time throughout life, without having discontinuities due to accidents or diseases"

The meaning of this postulate expresses the individual survival of the species in relation to the environment, and it defines the life concept, in which its property expresses the capability of living in terms of probability $(P(t))$ which grows continuously with time, from birth to death. For any $t_{2}>t_{1}$, it has

$$
P_{2}\left(t_{2}\right)>P_{1}\left(t_{1}\right)
$$

The probability of individual survival $(P(t))$ describes the search of resources, optimization of pathways, niche improvement, defence, and escape actions, and includes the chance of reproduction. Equation (2.1) is an expression of the use and optimization of the structure itself (capabilities) and the exploitation of existing resources. Indicates the effect of learning on the environment, that is, the biological systems know their environment and give it the versatility to live. 


\subsection{Model of Surviving}

The probabilistic concept is used when the actions of the living beings are integrated into a memory structure to later use it as an advantage, and thus increase the probability of living.

In this work, we assume that the probability of individual survival is a monotonic function of time $(t)$, which suddenly goes to zero at the time $T$ when the characteristic lifetime of a biological species is reached.

Furthermore, it is assumed that any biological system is trapped during a period of well-being, and then released due to needs for protection, feeding or activities to compensate for changes in the environment. This implies a fractal Lévy walk behaviour [19].

With these two assumptions, the probability of survival $(P(t))$, while the system remains alive without accidents or diseases, can be expressed as:

$$
P\left(\frac{t}{T}\right)=A\left(\frac{t}{T}\right)^{\alpha}+B
$$

Valid for $0 \leq t \leq T$

According to the assumptions the power law model of Equation (2.2) follows the fractal kinetics [20] [21] with $0<\alpha<1$.

Equation (2.2) denotes the state of vitality of a representative member of the species at the time $t$. The vitality capacity of the prototype is contained into the information of the parameters $T$ and $\alpha$. The constant $B$ is also defined by the initial probability as $B=P(0)$, protection at birth.

In fact, $T$ is characteristic of a community of species in a given environment, integrating their interaction with it, which is translated into information and is part of the system. The parameter alpha $(\alpha)$ represents the information of the gene pool revealed in the autopoiesis activity [22].

We make a complete formulation when the probability is normalized to one,

$$
\int_{0}^{1} P\left(\frac{t}{T}\right) \mathrm{d}\left(\frac{t}{T}\right)=A \int_{0}^{1}\left(\frac{t}{T}\right)^{\alpha} \mathrm{d}\left(\frac{t}{T}\right)+\int_{0}^{1} B \mathrm{~d}\left(\frac{t}{T}\right)=1
$$

The result is the following,

$$
\frac{A}{\alpha+1}+B=1
$$

The constant $A$ must be expressed in terms of the gene pool parameter ( $\alpha$ ) and the initial probability. Therefore, in the model there are three free and independent parameters, which must be determined due to the lack of information on any species ( $\alpha, B$ and $T)$. The probability of survival always increases except when there is an accident or a disease, in which the probability could decrease but, if there is life, the property of monotonous growth is maintained and the probability will rise to the extent of the circumstances.

The members of a species are always working to keep their survival high and preserve life (struggle for life). The search for the highest probability of survival eventually derivatives from aggressive [23] or cooperative [24] social behaviour, 
and the results obtained with advantage are illustrated in the first characterize of life. The dependence of probability of survival regardless the reduction of the time variable $(t / T)$ including the genetic factor for several values of parameter $(\alpha)$ is shown graphically in Figure 1.

The result of the established survival model shows that, regardless of the animal species, given a pair of parameters $\alpha$ and $B$, and the normalized probability $(P)$ of survival (representative of organic and reproductive maturity), the same life expectancy is achieved in half the time (see colour lines and inset in Figure 1). This means that the development of an animal species in a given environment has achieved the same probability of survival in the middle of its expected life, including its reproduction.

\subsection{Survival Surpassed by Death: Aging Effect}

The model described above corresponds to the ideal case, in which the aging process does not occur, and death occurs accidentally. In contrast, and according to Bortz II [25], aging should be included to show that the probability of survival is reduced by the loss of physiological abilities [26]. This suggests the modification of Equation (2.2) [27] and it is proposed to add a fractal evolution term decreasing the ideal probabilities in the following way

$$
P(t)=P_{1}(t)+P_{2}(t)
$$

where the first term is the probability initially proposed in Equation (2.2), still valid for $0 \leq t \leq T$. Now, it can be called living probability.

The second term in Equation (2.5) represents the decrease in the probability of survival, or better known as the probability for death, due to aging. We assume that this starts at the critical time $t_{0}$ and it can be expressed as a negative function.

$$
P_{2}(t)=-D\left(\frac{t-t_{0}}{T}\right)^{\beta}
$$

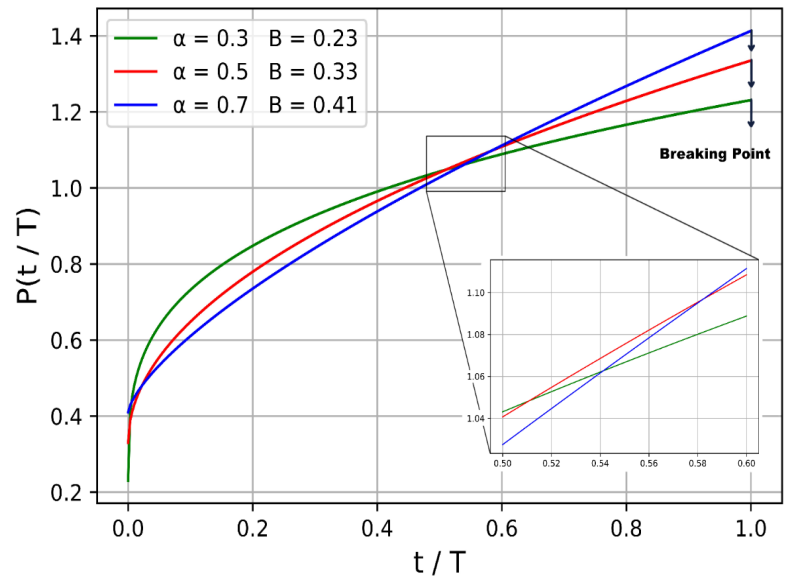

Figure 1 . The model of living probability per the formulation of Equation (2.2) is presented. The black arrow at $t / T=1$ represents the breaking point when death occurs. 


\section{Valid for $t_{0} \leq t \leq T$}

where $t_{0}$ is the starting time of the effects of aging and $\beta$ is the fractal exponent associated with damage, different from $\alpha$, since the exponent $\beta$ depends more on the living conditions and metabolism than on the genetic load [28]. In terms of simplicity, $D=A$ indicates that aging has the same statistical weight as individual survival.

Particularly, equation (2.6) does not necessarily describe death as a case in which $P(t)=0$ at $t=T$. Death is still accidental or caused by the inability to survive, the whole organism is no longer sustained.

Note that, if $t_{0}$ equals zero it would indicate that the aging process starts from birth. In this case, we could consider that a damaged organ or tissue was already present at a given time [29]. For simplicity and to separate the effects of the two processes described in Equation (2.6), the starting time is considered as

$$
t_{0}=\frac{T}{2}
$$

Now, the survival curve would show a maximum around $t_{0}$ as it is shown in Figure 2.

It should be noted that the presence of aging does not violate the first statement, which guarantees the growth of survival probabilities. Explained in this way: the life support provided primarily by inheritance is diminished by mutation or disease.

We would say that life has been defined as the continuity in health that sustains physiological functions. The aging process [30] grows at the expense of the reduction in the number of healthy cells until it causes the break point, when it is no longer possible to keep the organism alive.

The survival growth argument in the first characteristic of life is supported by cloning [31], cryopreservation of organs and cells [32] and anhydrobiosis [33]. Stating that aging destroys the effect of life but does not eliminate the inherent vital property that biological matter possesses.

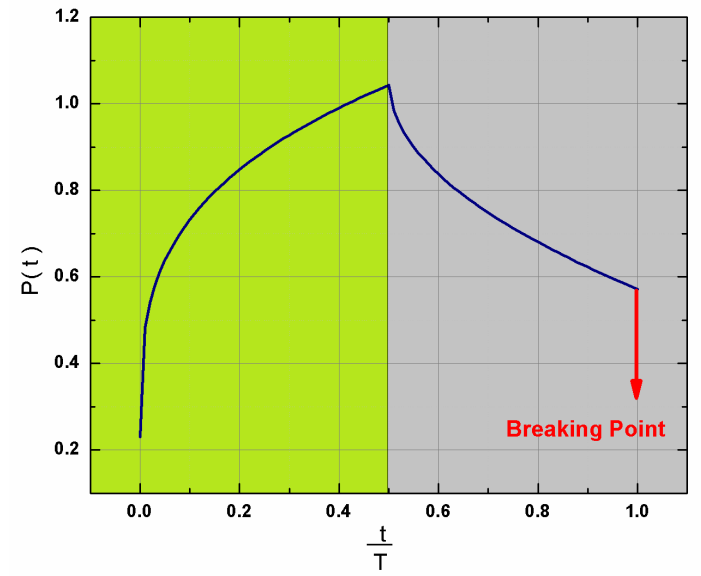

Figure 2. Result of Equation (2.6) with the condition of Equation (2.7) and using the parameters $\alpha=0.3$ and $\beta=0.6$. The ascendant line of life and the late descending line of aging and death are shown. 


\subsection{Activation Entropy Associated to the Surviving Probability}

The first characteristic of life is related to the activation entropy $(\Delta S)$, defined according to the relation

$$
P(t)=B \mathrm{e}^{\frac{\Delta S}{k}}
$$

where, $k$ is a representative constant. The activation entropy $(\Delta S)$ is equal to

$$
\Delta S=S-S_{0}=k \ln (P(t))
$$

where $P(t)$ is defined as before.

The reference of $S$ is given at $t=0$, where $P(t=0)=B$, then $S_{0}=k \ln B$.

The meaning of $S$ is established in the theory of fluctuations of thermodynamics [34] and represents the entropy that must be produced in each step of the physiological processes to maintain life, in parallel to the requirements of metabolism. In this way, we can say that the activation entropy of life is associated with the increased vitality.

\section{Second Characteristic of Life: Adaptive Evolution}

We consider heterotrophic organisms as open systems with thermal interactions and exchange of matter with the environment. The control of the internal state is carried out through cybernetic mechanisms, which means that the internal state can vary to adjust within certain limits to the environment. We will refer to the clinical parameters of normality that originate a health interval, or physiological intervals of variation.

Stability, an important characteristic of adaptation, is defined as the ability of the system to return to operating conditions in the range of clinical parameters when they have been altered by internal or external disturbances.

\subsection{General Vision of the Second Characteristic}

One of the possible conditions of biological evolution could be to maintain a steady state of minimum entropy production independent of the external environment. Sudden variations in external environments play an important role as they can alter clinical parameters and put the stability of organisms at risk. The search for a return to a stable state is possible through the internal control of organisms and efforts will be made to achieve a similar stable state.

In this sense, it is possible to consider the evolutionary transit within a development to avoid internal chaos [35] [36]. To supress that, evolution tends to establish changes in biological systems. Consequently, it is postulated that the development of organs occurred by natural selection in the search to organise physiological functions around stable internal states.

A biological system is related to its environment generating a nest that protects it against environmental changes and at the same time allows its adaptation by operating as a thermostat (regulator of vital thermodynamic parameters around clinical values or intervals). The meaning of the operation of a thermostat is taken in the broadest physiochemical sense from R. Kubo [37], who cited 
Bridgeman's work [38] when he said that: "Life then appears in some way to oppose the otherwise universal drive to disorder. Not only internal but also external forces should be present to enhance energy production inside the biological systems, e.g., metabolism. Periodic oscillations of the environmental parameters around the mean values play the role of such forces. Let us stress that the essential periodically changing external factors are an inseparable thermodynamic effect of the environment on the evolution of the biological system [39]. Hence, the joint effects of internal thermodynamic factors (expressed inside the system) and external thermodynamic effects (changes and oscillations of the environmental physical parameters) determine the direction of evolution".

We consider that the adaptation of an animal species is established when the living conditions are achieved in the proximity of a stable state with a minimum production of entropy, according to the external conditions.

"The necessary and sufficient condition for survival-adaptation is to keep physiological operations close to a state of minimum entropy production, compatible with environmental conditions"

The logical content of this statement is conditional, if a biological species achieves adaptation, it ensures that it is operating in the vicinity of a steady state of minimal metabolism according to the genotype and environment. On the contrary, if the latter is not achieved, there would be no adaptation and the species remains endanger of extinction. The underlying mechanism of adaptation is predicted to be natural selection.

We will illustrate the previous statement with the following model of evolution of the skin of homeothermic vertebrates.

\subsection{Physical Description of the Model}

The heat flow through the skin connects the transfers of thermal energy from the interior to the exterior of living organisms. In the case of homothermic animals (warm-blooded animals) such as mammals and birds, the internal temperature is maintained through the internal energy dissipation mechanisms, independent of the temperature of the environment within certain limits. For example, the internal temperature of the human being oscillates between $28^{\circ} \mathrm{C}$ and $36^{\circ} \mathrm{C}$ without any detrimental effect on the organic system.

A homeothermic organism has the need to transmit heat to the external environment to keep the internal temperature constant. This energy transfer makes it a dissipative structure [40]. The means of transfer are radiation (due to the difference in exterior and interior temperatures), convection (respiration) and conduction (through the skin). This section focuses on the heat loss from the organic interior through the skin. Figure 3 shows a diagram of the temperature distribution around the skin.

The skin layers (covers) of different constitutions acquired by vertebrates such as hair, feathers and shells, represent a protection against the convection of the environment around the epidermis considered as the coat or buffer of the wind chill effect. 


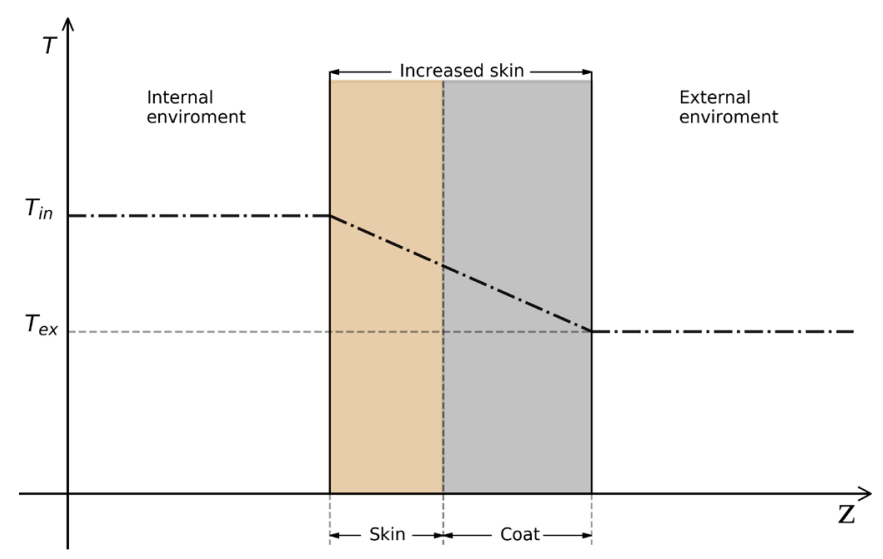

Figure 3. The coat with which animals cover the surface of the body has the effect of increasing the thickness of the skin. The interface composed of skin and coat is shown.

Biological evolution has led to the establishment of an organic structure in the skin to aid in the performance of exothermic function along with changes in the metabolic rate and diet. Currently, the skin of higher vertebrates with thermal homeostasis is a system composed of three diffusive membranes, the dermis, epidermis and hypodermis, each with different thermal conductivities. The skin integrated system has an effective thermal conductivity, which will be considered as constant for all purposes, depending only on the genotype.

The skin is the thermal barrier that separates two calorific reservoirs, the first one is the internal environment at the temperature $T_{i n}$ and, the second is the external environment at a temperature $T_{e x}$. Now, a cross section of unit area has the same constant temperature, which changes from the inside to the outside. Heat flow is considered only in the perpendicular direction to the cross section, since the considered section can be taken as isolated, and there are no lateral flows, the temperature distribution profile is linear. The amount of heat transmitted depends on the temperature gradient, that is, the external and internal temperature difference along the thickness of the skin along the $z$ axis. According to Fourier's law, heat flux depends on the temperature variation in a linear relationship between the flow and the thermodynamic force, which is expressed in terms of the temperature gradient $T$.

$$
q=-k \frac{\mathrm{d} T}{\mathrm{~d} z}
$$

where $k$ is the effective thermal conductivity, a parameter considered constant for the skin of a species in the time of exposure to the environment considering an experiment of a certain duration.

With these assumptions, the above flow-force relationship gives rise to steady state of minimum entropy production, and the steady state is stable against fluctuations. Conclusions are detailed below.

It is established that the entropy production $(\sigma)$ expressed by the product of the heat flow and the thermodynamic force is given by 


$$
\sigma_{s}=q \frac{\mathrm{d} T}{\mathrm{~d} z}>0
$$

The total production of entropy $(P)$ in the system (Lyapunov's function of a dynamic system) is the integral in the entire volume $(U)$ surrounded by skin,

$$
P=\int \sigma \mathrm{d} U=\int q \frac{\mathrm{d} T}{\mathrm{~d} z} \mathrm{~d} U
$$

The minimization of this function is achieved by varying the total entropy production (fluctuations of internal or external origin) and equalling to zero, which leads to

$$
\frac{\mathrm{d} q}{\mathrm{~d} z}=0
$$

Which putting in the energy balance equation leads to the following result [41]

$$
\frac{\partial T}{\partial t}=0
$$

Assuming there is a stationary state, in which the profile of internal and environmental temperature does not change. This shows that the minimum entropy production is associated with a steady state.

Following with the second part of the proof of principle of least entropy, the stability of the steady state will be demonstrated. See the verification of the minimum energy principle in the Appendix A.

$$
\frac{\partial P}{\partial t}=-2 \int \rho \frac{c_{v}}{T^{2}}\left(\frac{\partial T}{\partial t}\right)^{2} \mathrm{~d} U<0
$$

Equation (3.6), shows the entropy production decreasing with time with a variation of the state, verifying the stability of a steady state with minimum entropy and minimum metabolism [42]. Note that the quantities in the integrand are all positive, which results in the variation in entropy production decreases with time. In terms of this theorem established that a thermodynamic system in a steady state is stable with a minimum entropy production.

Now, we will illustrate how in the presence of external convection the validity of this principle is maintained by lowering the temperature, but its breaks down before freezing,

Consider Fourier's law applied to the skin in the absence of a convective effect (see curve (a) in Figure 4) the heat flux from the interior to the environment is established by

$$
q_{1}=\frac{k_{e f}}{L_{0}}\left(T_{i n}-T_{e x}\right)
$$

In the presence of the wind chill effect, the internally produced heat flow increases when the external temperature decreases as a function of the wind speed $V$, observing an equivalent external temperature $T_{e x}(V)$, where the heat flow (see curve (b) in Figure 4) is expressed as 


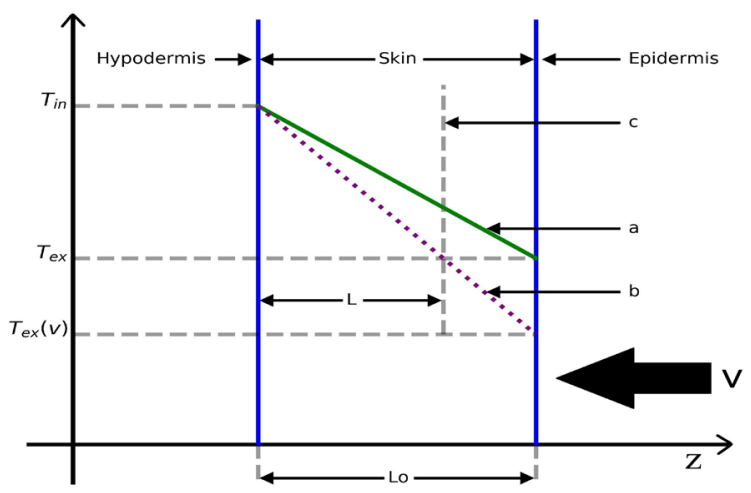

Figure 4. The initial skin thickness variation is shown $L_{0}$ at the effective thickness $L$ when there is a convective effect of air current in front of the skin. By decreasing the effective thickness of the skin, the amount of heat transferred increases from the inside to the outside of the body.

$$
\begin{gathered}
q_{2}=\frac{k_{e f}}{L_{0}}\left(T_{i n}-T_{e x}(V)\right) \\
q_{2}>q_{1}
\end{gathered}
$$

On the other hand, a linear temperature profile is maintained throughout the skin due to the generation of a dynamic change introduced by apparently reducing the thickness of the skin.

The following model exemplifies how the thickness of the skin is reduced, as we will see below. Considering the same amount of heat that is produced to maintain the internal temperature when observing that the initial external temperature $\left(T_{e x}\right)$ has penetrated to a certain depth due to the effect of the wind,

$$
q_{2}=\frac{k_{e f}}{L}\left(T_{i n}-T_{e x}\right)
$$

The effective thickness of the skin is $L$ and not $L_{0}$. That is, the difference in the thickness of the skin stops working to counteract the cooling.

By matching the two last Equations (3.8) and (3.10) the effective thickness of the skin operating in the presence of the wind chill effect is given by $L$ (see dotted line (c) in Figure 4) is

$$
L=L_{0}(1-x)
$$

where

$$
x=\frac{T_{e x}-T_{e x}(V)}{T_{i n}-T_{e x}(V)}
$$

here is a closer look at the limit cases of $x$. First, it is zero when the wind speed is zero and $T_{e x}=T_{e x}(V=0)$. Second, when $x \rightarrow 1$, this limit is not reached, since a decreased in internal temperature occurs due to the arrival of white noise (chaotic behaviour), and freezing can occur, causing the situation that $T_{i n} \rightarrow T_{e x}$. For illustration purposes, consider $T_{e x}=10^{\circ} \mathrm{C}$, wind at $40 \mathrm{~km} / \mathrm{h}$, and the equivalent temperature (wind chill [43]) is the freezing [44] (frostbite) one equal to $T_{\text {fros }}=-27^{\circ} \mathrm{C}$. Using the values in Equation (3.12) for Kelvin temperatures, it 
gives $x=0.58$. In this example we can see that since the freezing temperature was reached, our model is not valid because the skin is no longer a thermal barrier.

We now consider $T_{e x}(V)$ as the one established in the Wind Chill Temperature (WCT) chart. This chart, with air temperature in ${ }^{\circ} \mathrm{C}$ and wind speed in $\mathrm{km} / \mathrm{h}$, expresses the equivalent temperature with the following equation [45]

$$
T_{e x}(V)=13.12+0.6215 T-11.37 V^{0.16}+0.3965 T V^{0.16}
$$

In this Equation (3.13) the three effects of heat transmission, conduction, convection and radiation, have been considered.

The relation of $L$ and $L_{0}$ according to the Equations (2.11) and (2.12) is given by

$$
\frac{L}{L_{0}}=\frac{T_{i n}-T_{e x}}{T_{i n}-T_{e x}(V)}
$$

Figure 4 shows the variables in the considered system. The layers of the skin are shown and how the dermis (skin) is protected by the epidermis and hypodermis.

Figure 5 shows $L / L_{0}$ versus the wind speed $(V)$ within the interval in which the organism manages to recover its normal internal temperature after cooling, by increasing the wind chill effect.

Figure 5 shows the relationship of the effective thickness of the skin in the presence of the wind chill effect with respect to the original thickness. This figure shows that the external temperature can penetrate up to $20 \%$ into the skin and the profile decreases. According to the reference of the effects of the convection of the wind against the skin, exposure to winds greater than 40 $\mathrm{km} /$ hour at a temperature of $-150^{\circ} \mathrm{C}$ produces, according to our model, a penetration into the skin of the external temperature at depths greater than $20 \%$ is life-threatening.

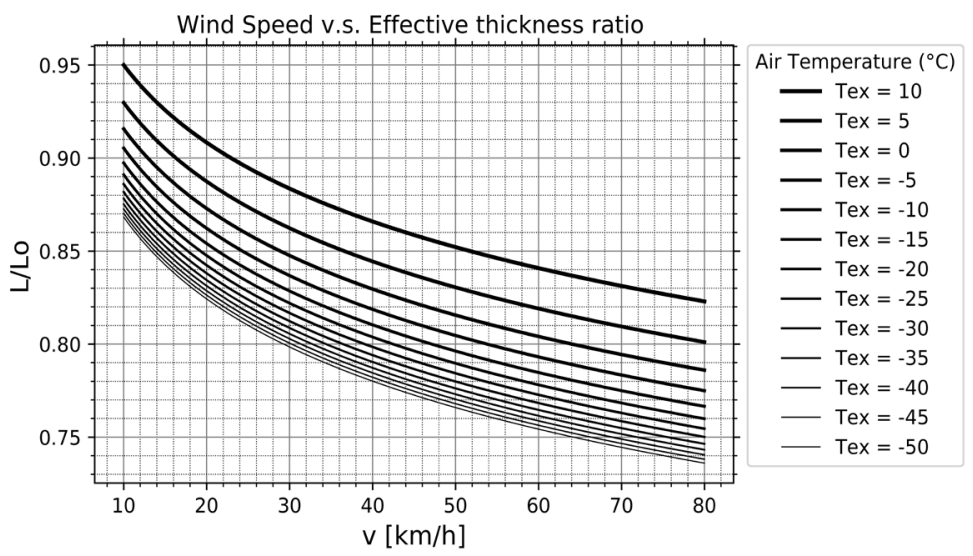

Figure 5. Ratio of skin thickness $L / L_{0}$ against wind speed for an air temperature at fixed values. There is a range of outside temperatures in the range $\left[-50^{\circ} \mathrm{C}, 10^{\circ} \mathrm{C}\right]$. Different lines are shown with a certain thickness for each temperature depending on the wind speed that is taken as constant (see vertical variation). As the wind speed increases, the separation between the lines in the graph decreases. 
In our model it is shown that in the presence of the wind the thickness of the skin decreases to a value $L$ [see Equation (3)]. An important appreciation at this point is that the external temperature and the thickness of the skin change, and the variation $L$ specifies the thermal sensation of cold. This is accomplished using equivalent temperature table Figure 6.

The behaviour of the dividing line between before and after freezing, depends on $V$ and $T_{e x}$. Linear regression offers the following equation

$$
\frac{L_{s}}{L_{o}}=A T_{e x}(V)+B
$$

where, $A=-0.0162$ and $B=0.5639$

Figure 7 qualitatively shows the change from the linear path to the non-linear, established by the limit $L_{s}$.

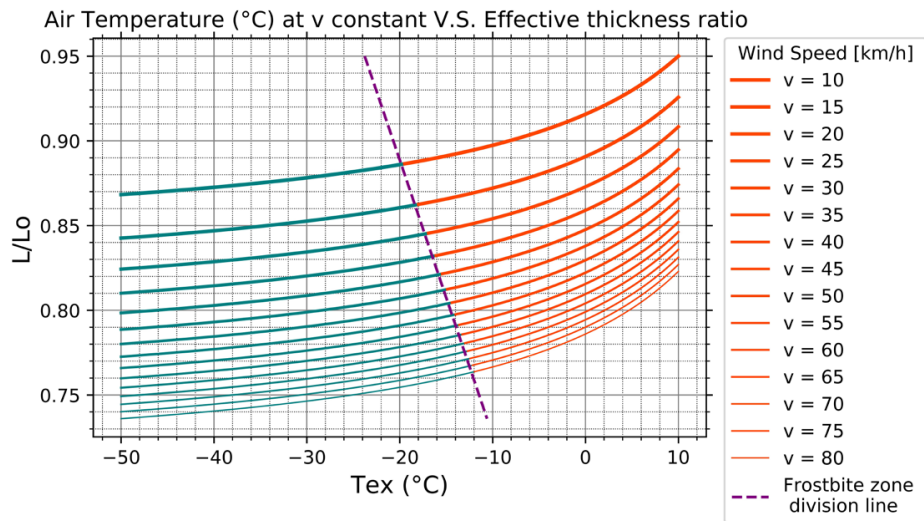

Figure 6. Frostbite zone division (see dashed line dividing the freezing zone) is the result of linear regression of the limit points before and after freezing for a constant wind speed.

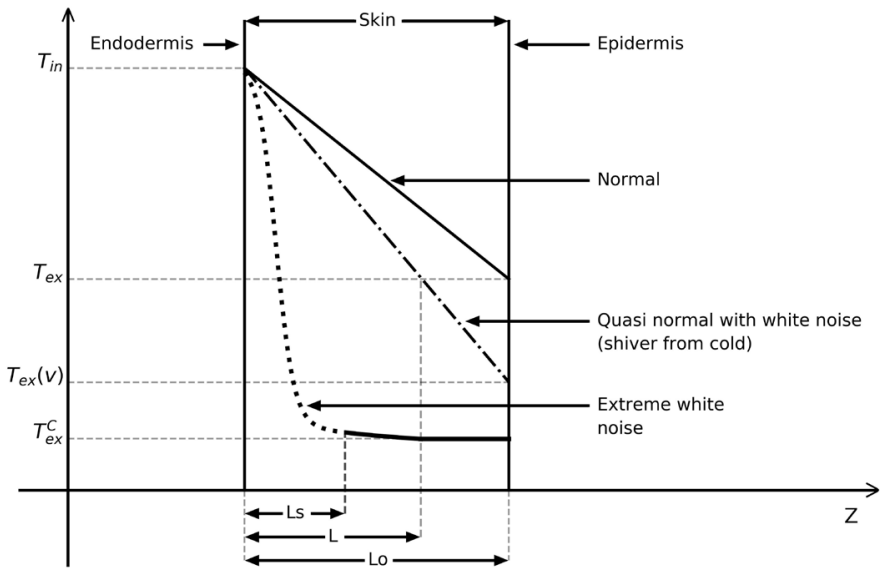

Figure 7. Different linear behaviour, normal (absence of wind) and quasi-normal (in the presence of wind). The dotted curve shows the non-linear dependence, which introduces the chaotic situation in the thermal behaviour at the freezing point. 
Figure 7 shows the effective length $\left(L_{s}\right)$ of the skin at the freezing point (see Equation (3.15). It is assumed that white noise occurs when the heat transport equation becomes stochastic [46], when $L$ changes for $L_{0}\left(L<L_{0}\right)$.

The limit of the change in $L$ remains at the freezing point denominated by $L_{s}$. According to the model presented, $L$ varies between the following limits.

$$
L_{s}<L<L_{0}
$$

Given that non-linearity (white noise) is accompanied by a state of stress, the evolution of the skin has been guided to protect it from the "wind cooling effect", achieving a linear temperature profile in the skin and stability against changes of temperature. This stability of the biological system has been integrated into physiological regulation by evolution. In other words, the evolution of biological systems has found a solution and can be exemplified in our model to show the effect of convection.

Based on this result, it is proposed body coverings of different constitutions that vertebrates have acquired [47], such as feathers and shells are explained by the search for an evolutionary trend that mitigates the effect of the wind cooling effect and brings the thermal regulation of homeothermic animals closer to the state of minimum entropy production.

\section{Third Characteristic of Life: Negentropy Principle}

This characteristic tells us about evolution whose description is well established in the synthetic theory [48] [49] [50]. Modifications are maintained in the hereditary characteristics of the DNA of biological species [51], but later some parts are eliminated by natural selection depending on the environment, while those that are conserved have an adaptive advantage [52] causing a biological-irreversible process in the evolution of the species. I. Prigogine [53] indicated that the main cause of evolution are the spontaneous fluctuations in the thermodynamic systems that later develop a deterministic character. In contrast, J. Monod [54] explained evolution as the continuous effect in terms of chance and necessity. Hence, the third statement of the evolution of life could be given as followed,

"The evolution of living matter is a persistent and biological-irreversible phenomenon"

1) Meaning of persistence.

It means that the mechanism of evolution does not stop acting and is always present regardless of whether it is by jumps or continuous, fast, or slow. It is ensured that, the rate of increase in average fitness of any organism is exactly equal to its genetic variability at that time [55], gradualism or discontinuous growth [56] (punctuated equilibrium principle). Therefore, the modification of biological structures through sudden changes in short times could seem as possible, as evidenced by artificial selection and experimental evolution using microbes [57] [58] [59]. Persistence explains speciation and the principle of divergence, that is, groups with more population turn out to be those that produce more species and 
subspecies, according to the fundamental description of natural selection [60]. Here, the capacity increases in different generations that seek adaptation in different directions are considered.

2) Explanation of the biological-irreversible:

Irreversibility means "arrow of time" and distinguishes the past from the future in genetically induced evolution and naturally selected in a given environment. For example, having established the evolution associated with changes in the environment, there would be a return to a previous environment that is surpassed by the species. The adaptation is not immediate, but the adaptive functions are restored to what has been accomplished, which indicates that evolution is not undone but on what has been achieved the new adaptation is established. Therefore, there is no inverse evolution if the change of environment is reversed, the previous functions are not recovered but a new adaptive process falls in the modified organs.

All living beings are structures in constant evolution, in which physical and chemical changes take place. Biological irreversibility is established due to these cumulative changes according to the trajectory of the species lifespan. It is possible to know the progress achieved, since living beings maintain memory through the modifications of their structures, but not through the transit of different environments and life forms (complementary are fossil and anthropological data). The genetic records represent the accumulated changes over time, which is inscribed in the inheritance, and from there the following modifications are made.

\subsection{Thermodynamic Formulation of the Principle of Negentropic Growth}

E. Schrödinger in 1944 [61] proposed the term "Negentropy" to indicate the property of biological matter to oppose a tendency to increase disorder and entropy, as stated in the second principle of thermodynamics. Instead, biological matter self-organises in the form of aperiodic structures with the possibility of genetically encoding the acquired structure in the DNA molecules [62].

Thermodynamically, the evolution of living matter is described by postulating the validity of the negentropy principle [63], which states that the evolution of biological system occurs in the direction [64] which the structure [65] becomes more complex. That is, the phylogenetic changes occur in the direction in which the negentropy of the structure increases.

In a biological system, the entropy after a series of evolutionary steps is $S_{1}$, and the change $\left(\Delta S_{1}\right)$ considering the reference of a totally disordered system with the same molecules $\left(S_{0}\right)$ is given as

$$
\Delta S_{1}=S_{1}-S_{0}
$$

Suppose that the same system $\left(S_{1}\right)$ continues to evolve, it will meet $S_{t}$ the entropy change regarding the previous state is

$$
\Delta S_{t}=S_{t}-S_{1}
$$

Negentropy is defined as the decrease in entropy that has accumulated over 
the course of successive generations.

$$
\Delta H=-\left(\Delta S_{t}-\Delta S_{1}\right)=S_{0}-S_{t}>0
$$

In the total evolution, a total change in negentropy has been gained, given by,

$$
\Delta H>0
$$

The $\Delta H$ is the negentropy intake (see Figure 8) and represents the gain in structure for a biological specie through its life history on earth that has taken about 3465 million of years [66].

Figure 8 shows the maximum entropy of a biological system, which depends on time according to its own morphogenetic evolution, recognised as phenotypic plasticity [67]. We take $S_{0}$ as a reference for the maximum entropy established by the total molecules that make up the biological body in the form of a simple mixture (homogenous system). As an example, a random set of molecules $\left(S_{0}\right)$ is taken. The entropy of a biologically structured (heterogeneous) system is $\left(S_{1}\right)$ which is a quantity that continues to decrease over time $\left(S_{2}\right)$ and so on $\left(S_{f}\right)$, (see dashed lines), and the total change is $\Delta H=S_{0}-S_{2}>0$.

Then $S_{1}$ is that entropy when the system has gained a certain structuring of the molecules to conserve and extend their life. That is, the dashed curve of $S_{1}$ is the survival line of a heterogeneous biological system and similarly, the dashed curve of $S_{2}$ and so on $S_{f}$. The continuous curve is the survival path of a heterogeneous biological system in a post evolution scenario.

\subsection{Negentropy Model}

From a thermodynamic point of view, genetic mutations are modelled representing a thermal fluctuation creating a genetic constriction. When a constraint is removed, the system spontaneously changes as in a free expansion (constant internal energy) and the entropy increases ( $\mathrm{d} S>0$ ). The opposite process establishes a constriction where the entropy decreases by which a mutation is established.

Consider a reversible process of a microsystem in contact with a heat reservoir at a constant temperature $T$. An external disturbance (could be a fluctuation) acts $\left(\delta W_{\text {rev }}\right)$ on the microsystem. In this case, the entropy change is negative ( $\mathrm{d} S$ $<0$ ), given by the equation

$$
\delta W_{\text {rev }}=-T \mathrm{~d} S=-\delta Q
$$

The term to the right of the equalities (rhs) is the amount of heat $(\delta Q<0)$ transferred to the surroundings so that the internal energy does not change. The total evolutionary work of a species is the repeated sum of the accumulated changes

$$
W_{\text {rev }}=-\int T \mathrm{~d} S=-T \Delta
$$

On the other hand, negentropy $(\Delta H)$ is given in terms of all accumulated works as

$$
\Delta H=\frac{W_{r e v}}{T}
$$




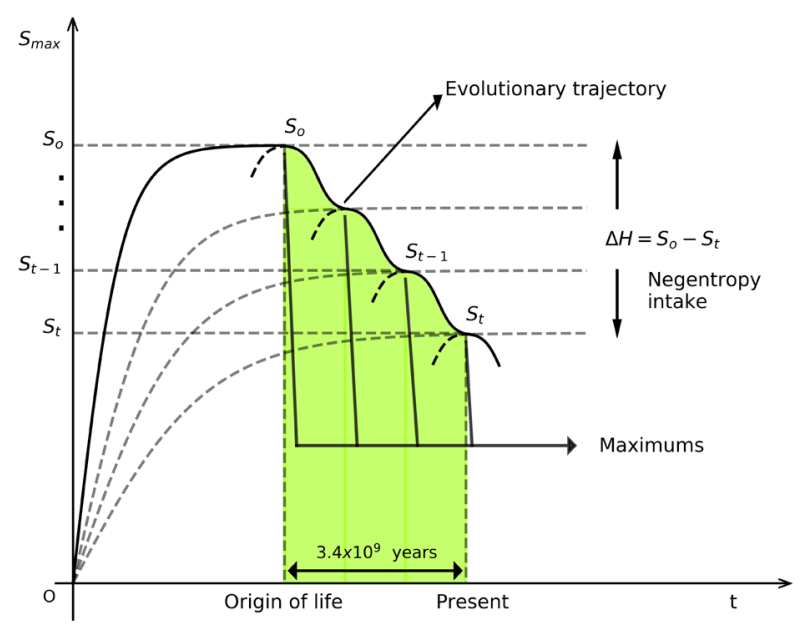

Figure 8. The maximum entropy is time dependent and the evolutionary trajectory is shown. The decrease in the maximum entropy of biological systems is established in evolution. Negentropy reflects this decrease and gives meaning to the gain in structure $\Delta H=S_{0}-S_{t}>0$.

and represents the degree of organization [68] established in the biological species.

The constant increase in this quantity $(H)$ indicates that the macroscopic process is biologically irreversible as the increase in the macroscopic and microscopic organization of DNA.

In terms of predictability, future events have several forms of occurring and depend on many fluctuations. However, past events are recorded and could be genetically determined, curiously in the direction in which negentropy increases. This can be seen in the validity of the description of the molecular clock hypothesis [69], phylogenetic trees [70] and evolutionary ladders [71].

\section{Conclusion}

We have established three characteristics of life in the form of postulates or statements and provided an illustrative example for each. The first postulate included the growth of the probability of survival, as a definition of life. In contrast to this process is aging introduced as a disturbance that is established in the system and increases permanently until the end of life. The second postulate covered the cybernetic regulation of biological systems, particularly homeothermic organisms. In this postulate, a possible tendency towards adaptation and stability was established. As part of this evolutionary development is homeostasis, maintaining clinical health parameters and establishing a stable state of minimum entropy production depending on the environment. The example provided for this characteristic was heat transfer and skin evolution for protection against the wind chill effect, in which a fit is ensured to maintain stationary clinical parameters. Finally, the third postulate incorporated the negentropy principle to ensuring that the direction of evolution is an incessant increase in organic structuring (biological-irreversible). The three postulates can be considered independent 
each other.

\section{Acknowledgements}

The authors would like to thank the projects UNAM-DGAPA-PAPIIT IG-100618 and IN 114818, and to Raúl Reyes Ortíz and Alberto López Vivas for the technical assistance. V. L-D is supported by Conacyt (Mexico) and the EPSRC grant (EP/ L016648/1) from the Bristol Centre for Functional Nanomaterials (University of Bristol).

\section{Conflicts of Interest}

The authors declare no conflicts of interest regarding the publication of this paper.

\section{References}

[1] del Castillo, L.F. and Vera-Cruz, P. (2011) Journal of Modern Physics, 2, 379-391. https://doi.org/10.4236/jmp.2011.25047

[2] Pogliani, L. and Berberan-Santos, M.N. (2000) Journal of Mathematical Chemistry, 28, 1-3. https://doi.org/10.1023/A:1018834326958

[3] Callen, H.B. (1985) Thermodynamics and Introduction to Thermostatistics. Second Edition, John Wiley and Sons, New York.

[4] Anokhin, P.K. (1974) Biology and Neurophysiology of Conditioned Reflex and Its Role in Adaptative Behavior. Pergamon Press, Oxford.

[5] Lindström, B., Selbing, I. and Olsson, A. (2016) PLoS ONE, 11, e0160245. https://doi.org/10.1371/journal.pone.0160245

[6] Sutter, M. and Kawecki, T.J. (2009) Journal of Evolutionary Biology, 22, 2201-2214. https://doi.org/10.1111/j.1420-9101.2009.01836.x

[7] Sznakder, B., Sabelis, M.W. and Egas, M. (2012) Evolutionary Biology, 39, 301-310. https://doi.org/10.1007/s11692-011-9155-2

[8] Prigogine, I. and Wiame, T.M. (1946) Experientia, 2, 451-453. https://doi.org/10.1007/BF02153597

[9] Bateson, P. (2017) Interface Focus, 7, Article ID: 20160126. https://doi.org/10.1098/rsfs.2016.0126

[10] Allen, C. and Bekoff, M. (1999) Species of Mind: The Philosophy and Biology of Cognitive Ethology. MIT Press, Cambridge.

[11] Emlen, S.T. (1995) PNAS, 92, 8092-8099. https://doi.org/10.1073/pnas.92.18.8092

[12] Eibl-Eibesfeldt, I. (1975) The Biology of Behavior, Holt, Rinehart and Winston. Second Edition.

[13] Altmann, J., Alberts, S.C., Haines, S.A., et al. (1996) PNAS, 93, 5797-5801. https://doi.org/10.1073/pnas.93.12.5797

[14] Lorentz, K. (1965) Evolution and Modification of Behavior. University of Chicago Press, Chicago.

[15] Bateson, P. (1991) The Development and Integration of Behavior: Essays in Honor of Robert Hinde. Cambridge University Press, Cambridge.

[16] Kalikow, T.J. (1983) Journal of the History of Biology, 16, 39-73. https://doi.org/10.1007/BF00186675 
[17] Krebs, J.R. and Davies, N.B. (1997) Behavioural Ecology. Blackwell Publishing Co., Hoboken.

[18] Shanor, K., Kanwal, J., Sing, B. and Giggle, M. (2009) Reviling the Secret Lives of Animals. Icon Books, London.

[19] Scher, H. and Montroll, E.W. (1975) Physical Review B, 12, 2455-2477. https://doi.org/10.1103/PhysRevB.12.2455

[20] Klafter, J. and Sokolov, I.M. (2015) Physics World, 18, 29-32. https://doi.org/10.1088/2058-7058/18/8/33

[21] Metzler, R. and Klafter, J. (2000) Physics Report, 339, 1-77. https://doi.org/10.1016/S0370-1573(00)00070-3

[22] Thomson, E. (2010) Mind in Life: Biology, Phenomenology and the Sciences of Mind. Harvard University Press, Cambridge.

[23] Wilson, A.J., de Boer, M., Arnott, G. and Grimmer, A. (2011) PLoS ONE, 6, e28024. https://doi.org/10.1371/journal.pone.0028024

[24] Axelrod, R. and Hamilton, W.D. (1981) Science, 211, 1390-1396. https://doi.org/10.1126/science.7466396

[25] Bortz II, W.M. (1986) Experimental Gerontology, 21, 321-328. https://doi.org/10.1016/0531-5565(86)90039-2

[26] Patrick, D.L. and Deyo, R.A. (1989) Medical Care, 27, 217-232. https://doi.org/10.1097/00005650-198903001-00018

[27] Harman, D. (1981) PNAS, 78, 7124-7128. https://doi.org/10.1073/pnas.78.11.7124

[28] Szilard, L. (1959) PNAS, 45, 30-45. https://doi.org/10.1073/pnas.45.1.30

[29] Curtis, H.J. (1963) Science, 141, 686-694. https://doi.org/10.1126/science.141.3582.686

[30] Strehler, B.L. (1977) Times, Cells, and Aging. 2nd Edition, Academic Press, New York.

[31] Rose, M.R. (1991) Evolutionary Biology of Aging. Oxford University Press, New York.

[32] Westhusin, M.E., Long, C.R., Shin, T., Hill, J.R., Looney, C., Pryor, J.H. and Piedrahita, J.A. (2001) Theriogenology, 55, 35-49. https://doi.org/10.1016/S0093-691X(00)00444-1

[33] Wolfe, J. and Bryant, G. (2001) International Journal of Refrigeration, 24, 438-450. https://doi.org/10.1016/S0140-7007(00)00027-X

[34] Buitink, J. and Leprince, O. (2004) Cryobiology, 48, 215-228. https://doi.org/10.1016/j.cryobiol.2004.02.011

[35] Katchalsky, A. and Curran, P.F. (1981) Non Equilibrium Thermodynamics in Biophysics. Harvard University Press, Cambridge, See Equation 15-18.

[36] Grebogi, C., Ott, E. and Yorke, J.A. (1987) Science, 238, 632-638. https://doi.org/10.1126/science.238.4827.632

[37] Sparrow, C. (1982) The Lorenz Equations: Bifurcation, Chaos, and Strange Attractors. Springer, New York. https://doi.org/10.1007/978-1-4612-5767-7

[38] Kubo, R. (1968) Thermodynamics. North Holland Pu. Co., Amsterdam, Divertissement 12 .

[39] Bridgeman (1943) The Nature of Thermodynamics. Harvard University Press, Cambridge.

[40] Collell, G. and Fauquet, J. (2015) Frontiers in Psychology, 6, 818. 
https://doi.org/10.3389/fpsyg.2015.00818

[41] Gladyshev, G.P. (1999) Entropy, 1, 9-20. https://doi.org/10.3390/e1020009

[42] De Groot, S.R. and Mazur, P. (1962) Non-Equilibrium Thermodynamics. North Holland Pu. Co., Amsterdam.

[43] Bluestein, M. and Zecher, J. (1999) Bulletin of the American Meteorological Society, 80, 1893-1899. https://doi.org/10.1175/1520-0477(1999)080<1893:ANATAA >2.0.CO;2

[44] Weinberg, A.D. (1993) Annals of Emergency Medicine, 22, 370-377. https://doi.org/10.1016/S0196-0644(05)80467-6

[45] Osczevski, R. and Bluestein, M. (2005) Bulletin of the American Meteorological Society, 86, 1453-1458. https://doi.org/10.1175/BAMS-86-10-1453

[46] Corona, O., Padilla, P., Escolero, O., Frank, A. and Fossion, R. (2013) Journal of Modern Physics, 4, 337-343. https://doi.org/10.4236/jmp.2013.43046

[47] Maderson, P.F.A. (2003) Experimental Dermatology, 12, 233-236. https://doi.org/10.1034/j.1600-0625.2003.00069.x

[48] Huxley, J. (1942) Evolution, the Modern Synthesis. George Allen \& Unwin, London.

[49] Eldredge, N. (1985) Unfinished Synthesis (Biological Hierarchies and Modern Evolutionary Thought). Oxford University Press, Oxford.

[50] Gould, S.J. (2002) The Structure of Evolutionary Theory. Harvard University Press, Cambridge.

[51] Crick, F. (1981) Life Itself: Its Origin and Nature. Simon and Schuster, New York.

[52] Huxley, J.S. (1942) Evolution: The Modern Synthesis. Harper, New York.

[53] Prigogine, I. (1983) Just an Illusion. Manuel Lugarte. (2008) The Laws of Chaos. First Spanish Edition, Drakontos Critica Editorial, Barcelona.

[54] Monod, J. (1970) Le hasard et la nécessité (Essai sur la philosophie naturelle de la biologie moderne) Paris.

[55] Provine, W.B. (1971) The Origins of Theoretical Population Genetics. University of Chicago, Chicago.

[56] Eldredge, N. and Gould, S.J. (1972) Speciation and Punctuated Equilibria: An Alternative to Phyletic Gradualism [3rd Draft]. In: Models in Palaeontology, Freeman, Cooper, and Co., San Francisco.

[57] Bull, J.J., Badgett, M.R., Wichman, H.A., Huelsenbeck, J.P., Hillis, D.M., Gulati, A., Ho, C. and Molineux, I.J. (1997) Genetics, 147, 1497-1507. https://www.genetics.org/content/147/4/1497

[58] Imhof, M. and Schlotterer, C. (2001) PNAS, 98, 1113-1117. https://doi.org/10.1073/pnas.98.3.1113

[59] Rozen, D.E., de Visser, J.A.G.M. and Gerrish, P.J. (2002) Current Biology, 12, 1040 1045. https://doi.org/10.1016/S0960-9822(02)00896-5

[60] Schrödinger, E. (1944) What Is Life? Mind and Matter. Cambridge University Press, Cambridge.

[61] Jacob, E.B., Shapira, Y. and Tauber, A.I. (2006) Physica A, 359, 495-524. https://doi.org/10.1016/j.physa.2005.05.096

[62] Ulanowicz, R.E. and Hannon, B.M. (1987) Proceedings of the Royal Society of London $B, 232,181-192$. https://doi.org/10.1098/rspb.1987.0067

[63] Ziehe, M. and Demetrius, L. (2005) Proceedings of the Royal Society B, 272, 11851194. https://doi.org/10.1098/rspb.2004.3032 
[64] Prigogine, I. (1978) Science, 201, 777-785.

https://doi.org/10.1126/science.201.4358.777

[65] Schopf, J.W. (1993) Science, 260, 640-646. https://doi.org/10.1126/science.260.5108.640

[66] Benbigh, K.G. (1989) The British Journal of the Philosophy of Science, 40, 323-332. https://doi.org/10.1093/bjps/40.3.323

[67] Nunney, L. (2016) Journal of Heredity, 107, 15-24.

https://doi.org/10.1093/jhered/esv084

[68] Maher, B.A. (2002) The Scientist, 16, 18.

https://www.the-scientist.com/research/uprooting-the-tree-of-life-52798

[69] Ciccarelli, F.D., Doerks, T., Von Mering, C., Creevey, C.J., Snel, B. and Bork, P. (2006) Science, 311, 1283-1287. https://doi.org/10.1126/science.1123061

[70] Fisher, R.A. (1999) The Genetical Theory of Natural Selection: A Complete Variorum Edition. Oxford University Press, Oxford.

[71] Fierst, J.L. (2011) Journal of Evolutionary Biology, 24, 1992-2001.

https://doi.org/10.1111/j.1420-9101.2011.02333.x 


\section{Appendix A}

According to the total entropy (expression 3.3) production is positive: $P>0$. Now we demonstrate that its variation over time is negative.

We start from the time derived of the total entropy with Equation (3.3) using Equation (3.1),

$$
\begin{gathered}
\frac{\partial P}{\partial t}=\frac{\partial}{\partial t} \int k\left(\frac{\mathrm{d} T}{\mathrm{~d} z}\right)^{2} \mathrm{~d} U \\
\frac{\partial P}{\partial t}=2 \int k \frac{\mathrm{d} T}{\mathrm{~d} z} \frac{\mathrm{d}}{\mathrm{d} z}\left(\frac{\partial T}{\partial t}\right) \mathrm{d} U
\end{gathered}
$$

Integrating by parts and considering that the temperatures at the edges of the skin remain constant, we have the following

$$
\frac{\partial P}{\partial t}=-2 \int\left(\frac{\partial T}{\partial t}\right)\left(k \frac{\mathrm{d} T}{\mathrm{~d} z}\right) \mathrm{d} U
$$

Employing the law of conservation of energy,

$$
\rho \frac{\partial u}{\partial t}=\rho c_{v} \frac{\partial T}{\partial t}=k \frac{\mathrm{d} T}{\mathrm{~d} z}
$$

where it has been considered that $\mathrm{d} u=\rho c_{v} \mathrm{~d} T$. Thus

$$
\frac{\partial P}{\partial t}=-2 \int \rho c_{v}\left(\frac{\partial T}{\partial t}\right)^{2} \mathrm{~d} U<0
$$

Note that the quantities in the integrand are all positive, which results in the variation in entropy production over time being a negative quantity, that is, decreases with time. In terms of the generalized Le Chatelier-Braun principle where it is established that the steady state is stable in the state of minimum production of entropy.

The conditions to give validity to this principle are that the thermal conductivity is constant and does not dependent on the conditions at the boundary. Furthermore, the linear relationship between the heat flux and the temperature gradient is maintained even with a variant ambient temperature. 